\title{
Oncogeriatrics (part 1.) Frailty in older adults with cancer
}

\author{
Jakub Kenig \\ Department of General, Oncological and Geriatric Surgery, Jagiellonian University Medical College, Kraków, Poland
}

\begin{abstract}
About onehalf of cancer cases and two-thirds of cancer deaths occur in patients 65 years of age or older. Therefore, understanding the health status of an older patient is just as important as staging of the cancer.

Frailty is a complex, multidimensional syndrome of increased vulnerability and loss of adaptive capacity/resistance to external stressors, resulting in an increased risk of adverse outcomes. Clinical presentation is non-specific: fatigue, unexplained weight loss, frequent infections, decline in physical mobility/balance/gait speed. Therefore, the routine format of preoperative investigations often does not allow for adequate frailty identification. There are two principal models of frailty: the phenotype model and the accumulation of deficits model. There is no consensus on an operational definition of frailty. However, it has been demonstrated that frailty, not chronological age, is the most important risk factor for poor outcome. Therefore, frailty identification should be obligatory before the beginning of the oncologic treatment.
\end{abstract}

NOWOTWORY J Oncol 2019; 69, 2: 55-57

Key words: older cancer patient, frailty definition, frailty symptoms, review

Similarly to other European countries, Poland's population is aging. In early 2030s, one-third of Poland's population will be aged 65 or older; in particular, the $80+$ group will double. There also is a gender imbalance at older ages. Almost $60 \%$ of older adults aged $70-74$ years are female and this proportion increases to 76\% for those 90-94 years old [1]. Additionally, taking the oncological context into consideration, about onehalf of cancer cases and two-thirds of cancer deaths occur in patients 65 years of age or older [2]. As the incidence of cancer increases with age, the number of elderly patients with cancer is expected to rise markedly in the next decade. However, this group of patients is very heterogeneous with regard to co-morbidity, physical reserve, cognitive function and social support. Studies report that $50 \%$ of patients over 70 years experience severe chemotherapy-related toxicity [3] and more than half have postoperative complications [4]. Therefore, identification of patients at risk is the key element for optimal and tailored treatment, particularly of cancer patients [5].
Frailty, by definition, is a complex, multidimensional, transitional state of increased vulnerability and loss of adaptive capacity/resistance to external stressors, resulting in an increased risk of adverse outcomes, both in the short and long term [5-7]. These external stressors comprise factors such as hospitalization, surgery, illness (even mild one in some frail patients) or environmental factors. In turn, adverse outcomes associated with frailty include: disability, hospitalization, postoperative complications, functional dependence, institutionalization, social withdrawal and death. In the context of cancer-specific treatment, it increases the risk of chemotherapy intolerance, disease recurrence and progression [8].

It is important to keep in mind that frailty is a concept, not an illness, and it is proposed to be used as a "clinical state variable", quantifying the underlying health status of the person [6].

In fit older adults, following a minor stressor, we observe a minor deterioration in function, followed by the return to homeostasis. In contrast, in a frail older patient a similar stres- 
sor causes a significant deterioration and, usually, without the return to the baseline homeostasis. This can lead to disability and death. Of note is that frailty is a dynamic state; in majority of population it will be chronic and progressing, however, in some patients it may be reversed to some extent [5].

The incidence of frailty is estimated to be between 10 and $20 \%$ of patients 65 or more of age and $25-50 \%$ of patients $85+$. However, the number depends on the assessed population and instruments used for it [9].

The etiopathogenesis of the frailty syndrome is complex and has not been fully understood. It is believed that a number of mechanisms may play a role: genetic factors, oxidative stress, mitochondrial dysfunction, chronic diseases that accelerate the development of the syndrome, lifestyle and the changes associated with the aging process [10]. Therefore, the frailty risk factors include: advanced age, polypharmacy, low physical fitness, cognitive disorders, malnutrition (low protein diet, deficits of multiple vitamins), poverty and isolation [10].

Frailty syndrome may not be evident to patients, family members, and - what is more important - to the untrained clinicians. Older people, who are classified as frail, frequently do not identify themselves as such [11]. By definition, manifestations associated within a syndrome occur in combination, and no single manifestation is sufficient to identify those with the syndrome. Clinical presentation is non-specific: fatigue, unexplained weight loss, frequent infections, decline in physical mobility, balance and gait impairment, delirium, fluctuating disability. Therefore, the routine format of, medical history, physical examination, biochemistry and imaging tests often do not provide the information needed for optimal identification of frailty. This is due that fact that it does not fit into classic organ-specific models of disease, there is a gradual decline in strength, endurance, and nutrition may not cause patients to seek medical attention and it often is attributed to old age [10].

Most of the physicians identify frailty with disability and/ or multi-comorbidity. Fried et al. examined the similarities and differences between those three factors. While there was a $21.5 \%$ overlap between the three categories, many more people were categorised into just one category or a mix of two categories. Only $6 \%$ of frail population was disabled (defined as being dependent in one or more activities of daily living), $46 \%$ had comorbidity (defined as the presence of 2 or more comorbid diseases), but - most importantly - $27 \%$ of frail population had neither poor function nor comorbidity [12]. Frailty is different conceptually from ageing, disability and co-morbidity, although it is related to these factors. Its prevalence increases with age, however, it occurs independently from chronological age [12].

The concept of frailty is still debatable and at present there are two principal models of it: the phenotype model described by Fried et al. [12] and the cumulative deficit model proposed originally by Rockwood [13]. The first model, widely used (though it has not been validated in older cancer patients), is based on the concept that a clinical syndrome driven by age-related biologic changes that drive physical characteristics of frailty and eventually, adverse outcomes. The frailty phenotype, introduced by Fried et al., was defined as meeting three or more of the following criteria: unintentional weight loss, self-reported exhaustion, slow walking speed, weak grip strength, and low physical activity level [12]. In turn, the basis of the deficit model is the accumulation of medical, functional, cognitive and social deficits that can be assessed using Geriatric Assessment and allowing to quantitatively summarize vulnerability. The research conducted in our centre showed that the model based on the accumulation of deficits had the highest predictive power in the preoperative evaluation of older patients with cancer. The sum of tools, not individual results, was an independent risk factor for 30-day mortality and postoperative complications. In turn, the number and type of incorporated instruments had a great influence on the prevalence of frailty and on adequate surgical risk assessment [14-15]. At present, we have good evidence that preoperative identification of frailty can forecast areas of vulnerability, treatment outcome (including morbidity and mortality), length of hospitalization and need for discharge to a skilled nursing facility. This knowledge allows for the tailored approach improving the treatment outcome and avoiding unnecessary harm, particularly to frail older patients.

\section{Conclusions}

Frailty is not a disease; it is a concept, unifying the care of older patients and directing attention away from organ-specific diagnoses towards a more holistic viewpoint of the patient. At present, there is no consensus on an operational definition of frailty which differs among authors and studies. However, there is more than good evidence that overtreatment in the frail patient can lead to unacceptable outcomes with high mortality or persistent disability. Moreover, in this population quality of life, functional decline and toxicity of the treatment is often much more important than prolonging of life alone. Therefore, identification of frailty should be obligatory before the beginning of the oncologic treatment.

This mini-review is the first part of the series, prepared by the Oncogeriatric Section of the Polish Society of Surgical Oncology, aiming the dissemination of knowledge on diagnostic and high-quality treatment of older patient with cancer.

Conflict of interests: none declared

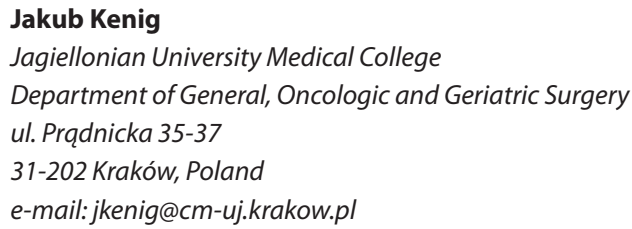

Received and accepted: 1 Jul 2019 


\section{References}

1. Eurostat. Mortality and life expectancy statistics. Retrieved from http:// epp.eurostat.ec.europa.eu/statistics_explained/index.php/Mortality and life expeancy statistics

2. Hurria A, Gupta S, Zauderer M et al. Developing a cancer-specific geriatric assessment: a feasibility study. Cancer 2005; 104: 1998-2005.

3. Extermann $\mathrm{M}$, Boler I, Reich RR et al. Predictingthe risk of chemotherapy toxicity in older patients: the chemotherapy risk assessment scale for high-age patients (CRASH) score. Cancer 2012; 118: 3377-3386.

4. Kristjansson SR, Nesbakken A, Jordhøy MS, et al. Comprehensive geriatric assessment can predict complications in elderly patients after elective surgery for colorectal cancer: a prospective observational cohort study. Crit Rev Oncol Hematol 2010; 76: 208-217.

5. Clegg A, Young J, Iliffe $S$ et al. Frailty in elederly people. The Lancet 2013; 381(9868): 37-44.

6. Rockwood K, Mitnitski A. Frailty defined by deficit accumulation and geriatric medicine defined by frailty. Clin Geriatr Med 2011; 27: 17-26.

7. Fried $\mathrm{LP}$, Tangen $\mathrm{CM}$, Walston $\mathrm{J}$ et al. Frailty in older adults: evidence for a phenotype. J Gerontol A Biol Sci Med Sci 2001; 56: 146-156.

8. Ethun CG, Bilen MA, Jani $A B$ et al. Frailty and cancer: Implications for oncology surgery, medical oncology, and radiation oncology. CA Cancer J Clin 2017; 67: 362-377.
9. Handforth C, Clegg A, Young C et al. The prevalence and outcomes of frailty in older cancer patients: a systematic review. Ann Oncol 2015; 26: 1091-1101.

10. Chen X, Mao G, Leng SX. Frailty syndrome: an overview. Clin. Intervention Aging 2014; 9: 433-441.

11. Grenier A. The distiction between being and feelinf frail: exploring emotional experiances in health and social care. Journal of Social Work Practice 2006; 20(3): 299-313.

12. Fried $L P$, Tangen $C M$, Walston J et al. Frailty in older adults: evidence for a phenotype. J Gerontol A Biol Sci Med Sci 2001; 56: 146-156.

13. Rockwood K, Mitnitski A. Frailty defined by deficit accumulation and geriatric medicine defined by frailty. Clin Geriatr Med 2011; 27: 17-26.

14. Kenig J, Zychiewicz B, Olszewska U et al. Screening for frailty among older patients with cancer that qualify for abdominal surgery. $J$ Geriatr Oncol 2015; 6: 52-59.

15. Kenig J, Zychiewicz B, Olszewska U et al. Cumulative deficit model of Geriatric Assessment to predict the postoperative outcome of older patients with solid abdominal cancer. J Geriatr Oncol 2015; 6(5): 370-379 